\title{
Endodontic tragedy- retrieval of unusual foreign body- a case report
}

\author{
Dr. AdnanAhmed, Dr. Nagalakshmi Chowdhary, Dr. MukundaKS, \\ Dr. KiranNK, Dr. AbhishekBansal, Dr. FehanAhmedKhan.
}

\begin{abstract}
The discovery of foreign bodies in the teeth is a special situation, which is often diagnosed accidentally. Sometimescomplications can occur during or after endodontic treatment, which may be due to negligence of the operator. According to a satisfactory obturation of the root canal, the filling material and the endodontic instruments should be limited to the root canal without extending to periapical tissues or neighbouring structures. This case report describes the successful retrieval of a button from the pulp chamber and gutta-percha lying beyond apex of the permanent mandibular first premolar.
\end{abstract}

Keywords: Foreign Object, Children, Management

\section{Introduction}

The main objective of endodontic treatment is to provide hermetic seal of the root canal system with an inert, biocompatible, and dimensionally stable filling material. ${ }^{1}$ It is generally agreed that all root canal filling materials should be confined to the root canal system. However, one complication that occurs during obturation is overextension, especially in cases of immature, resorbed or overinstrumented root canal apices. ${ }^{2}$ Foreign bodies inside tooth are diagnosed accidentally on clinical or radiographic examination of the tooth which may be associated with infection, pain and swelling. ${ }^{3}$

It is essential that the dentist takes a thorough history and performs a detailed examination, including an appropriate radiographic examination. ${ }^{4}$ These objects can be retrieved with some ease if they are located within the pulp chamber, but once has been pushed apically retrieval may be more complicated. ${ }^{5}$ This case describes the overextension of filling material from the apical foramen of the root canal due to operator's negligence and also presence of unusual foreign body in the root canal and their retrieval.

\section{Case Report}

A 13 year old boy reported to the department of pedodontics and preventive dentistry, sri Siddhartha dental college and hospital, with a chief complaint of pain and swelling in the lower left back tooth region from the past 15 days. His medical history was insignificant. Patient had undergone root canal treatment of 35 from private dental clinic 1 year back. Extra oral examination showed slight swelling in the left mandibular premolar region. Radiographic examination of the tooth revealed a radioopaque object lying beyond the apex which was about $3 \mathrm{~mm}$ in length. OPG was performed to confirm the location of the foreign object.Patient was advised to take antibiotic and analgesic. After routine blood investigation and taking clinical and radiographic findings into consideration, it was decided that extraction should be initiated, with an attempt to retrieve the foreign object.

Finally patient was recalled for the extraction of the tooth. After giving local anaesthesia, the gingival tissues was retracted using periosteal elevator. The crown portion of the tooth was removed first to locate the foreign body. As a surprise, button was present in the pulp chamber which was removed using locking twizzer. Patient was unaware of the entry of button in the pulp chamber. After retrieval of the button, the root portion was removed. Gutta-percha lying beyond the apex was later removed using locking twizzer. The object was identified as no- 25 gutta-percha point. After successful removal of the tooth and retrieval of the foreign body, suturing was done.

After 1 week, sutures were removed and the patient was found to be totally asymptomatic and socket was also in healing stage.

\section{Discussion}

Gutta-percha has been the most widely used root canal filling material for over 100 years. Knowledge of its composition, properties and behaviour has grown. Most marketed gutta-percha points contain about 19\% to $22 \%$ gutta-percha, $59 \%$ to $75 \%$ zinc oxide, $1 \%$ to $17 \%$ heavy metal salts and $1 \%$ to $4 \%$ wax or resin. ${ }^{6}$ One of the most iatrogenic complications in endodontic is overfilling of the root canal, which has a negative effect on prognosis of endodontically treated teeth (Brkicet al.; 2009). More than a half of the overfilled teeth heal satisfactorily after proper endodontic therapy, but in case of injury of any nerve or presence of obturating material in soft tissues or sinus spaces a surgical approach is necessary (Brkicet al.; 2009). ${ }^{1}$ Aclinician must, 
therefore, carefully measure and obturate canal systems using apex locator devices and radiographs to confirm working lengths. ${ }^{6}$

In the case described above, there was over extension of the root canal filling material that had cause the foreign body reaction and also placement of the foreign body (small button) by the child patient unknowingly.

Various foreign objects were reported to be lodged inthe root canals and the pulp chamber, which rangedfrom pencil leads, darning needles, metal screws, tobeads and stapler pins. Retrieval of foreign objects lying in the pulp chamberor canal can be done using simple method using needleby engaging it with instrument and pulling it outor by the costly instruments like ultrasonic instruments, theMasserann Kit, etc. Steglitz forceps is a specializedinstrument with a narrow beak used to retrieveinstruments from the coronal portion of canal. ${ }^{7}$ Nehme has recommended the use of operating microscope along with ultrasonic filing to eliminate intracanal metallic obstructions. ${ }^{8}$ Srivastava \&Vineeta have suggested periapical surgery or intentional reimplantation to remove such objects. They reported retrieval of a straight pin lodged in the periapical area of maxillary central incisor by periapical surgery. ${ }^{9}$

According to the American Dental Association, overfilling by more than $2 \mathrm{~mm}$ past the radiological apex represents a technical error ascribable to overinstrumentation, inadequate measuring, or a lack of an apical stop.

Over instrumentation, in particular, may extrude infected material contained in the canals beyond the apex, interfering, or impeding the healing process of the periapical tissue. The consequences of overfilling can, therefore, result in periapical infection caused by the transport of bacteria beyond the apex and an incomplete cleansing, foreign body reactions, and pain symptoms. ${ }^{1}$

Finally, the prognosis for an endodontically treated tooth with overfilling depends on the response of the periradicular tissue to the canal obturation material which is, in its own way, a consequence of the complexand, at times, an unpredictable interaction between the materials and the host defences. ${ }^{10,11}$

\section{Conclusion}

The clinician must practice careful and judicious shaping strategies that use multiple confirmations of working length and take serious precaution against overinstrumentation. It is important to use 'resistance form' in controlling overfills. This 'resistance form' can be impartedduring canal preparation by producing funnel form, tapered preparations and by selecting gutta-percha cones to match those canal shapes which will resist the obturation forces which promote extrusion.

This case describes the retrieval of foreign body as well as over obturatedgutta-percha from the tooth. Our main concern is to protect patients from harm when we as a professional can provide advanced and sophisticated therapies in a safe and controlled manner with patient safety as a foremost priority.

\section{References}

[1]. Rakesh K Y, SharadC, PromilaV, AnilC, AseemP K, KulwinderK W. Clinical negligence or endodontic mishaps: A surgeons dilemma. Natl J MaxillofacSurg 2012 Jan-Jun; 3(1): 87-90.

[2]. Silva EJNL, Herrera DR, Lima TF, Zaia AA. A nonsurgical technique for the removal of overextended gutta-percha. J Contemp Dent Pract 2012; 13(2): 219-221

[3]. Dhull KS, Acharya S, Ray P, Dhull RS. Foreign body in root canals of two adjacent deciduous molars: A case report. Int J ClinPediatr Dent 2013; 6(1): 38-39.

[4]. N. Mcauliffe, N. A. Drage, B. Hunter. Staple Diet: A Foreign body in a tooth. International Journal of Paediatric Dentistry $2005 ; 15: 468-471$.

[5]. Aduri R, Reddy RE, Kiran K. Foreign objects in teeth: Retrieval and management. J Indian SocPedod Prevent Dent Jul-Sept 2009; 3(27): 179-83.

[6]. Mahmoud R.E, Haskell T.D, Catherine F.P. Surgical Resolution Of Chronic Tissue Irritation Caused By Extruded Endodontic Filling Material. J Can Dent Assoc 2005; 71(7):487-90.

[7]. Pratima S, Archana K, Mohit G. Simple Means Of Management Of Teeth With Foreign Body In Root Canal. Indian Journal of Multidisciplinary Dentistry, 2011 Jan-Feb; 1(2): 78-81.

[8]. Nehme WB. Elimination of intracanal metallic obstructions by abrasion using an operational microscope and ultrasonics. $\mathbf{J}$ Endod. 2001 May;27(5):365-7.

[9]. Srivastava N, Vineeta N. Foreign body in the periradicular area. J Endod. 2001 Sep;27(9):593-4.

[10]. Gutierrez JH, Brizuela C, Villota E. Human teeth with periapicalpathosis after overstrumentation and overfilling of the root canals: A scanning electron microscopic study. IntEndod J 1999;32:40-8.

[11]. Nygaard-Ostby B, Hjortdal O. Tissue formation in the root canal following pulp removal. Scand J Dent Res. 1971;79(5):333-49. 


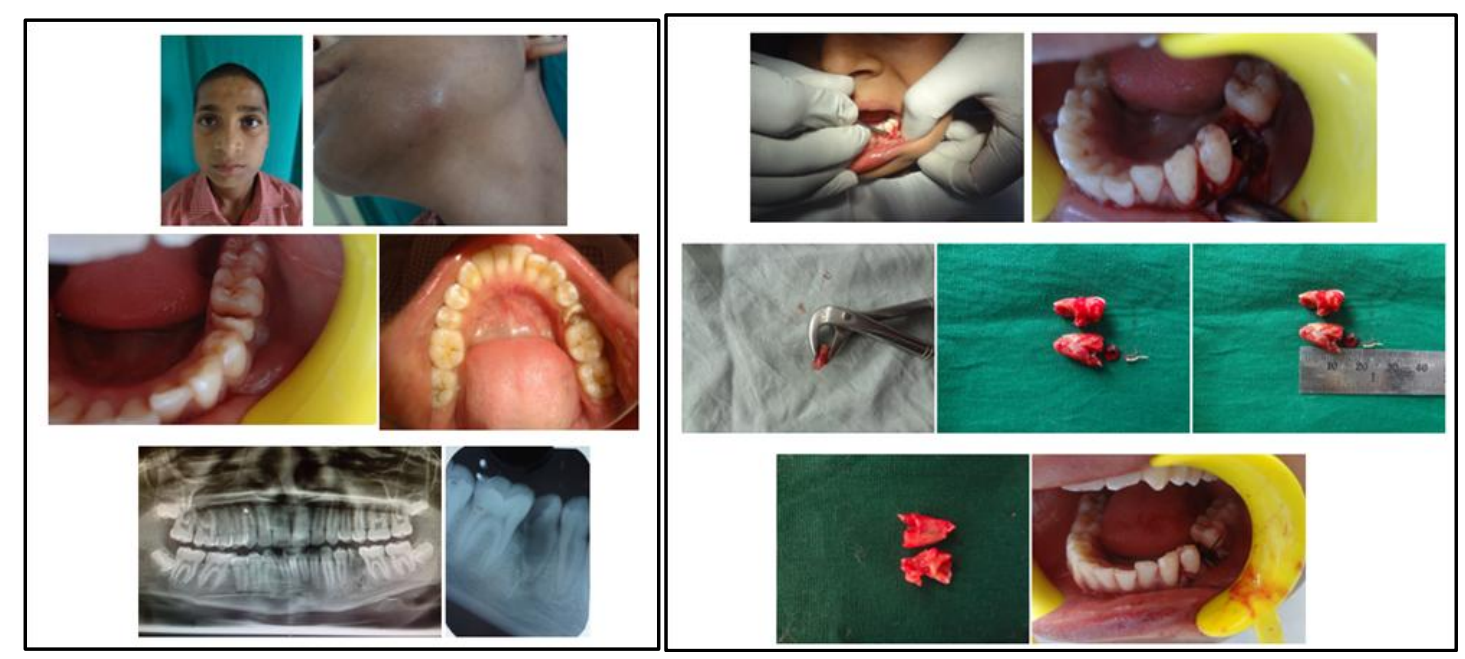

Pre treatment

Post treatment 